\title{
Inquinamento atmosferico e alterazioni scheletriche
}

\author{
Silvia Migliaccio ${ }^{1}$
}

Accettato: 27 settembre 2020 / Pubblicato online: 11 novembre 2020

(c) Springer Nature Switzerland AG 2020

\section{Commento a:}

Molecular and cellular mechanisms linking air pollution and bone damage.

D. Prada, G. López, H. Solleiro-Villavicencio,

C. Garcia-Cuellar, A.A. Baccarelli.

Environ Res (2020) 185:109465

È noto come l'inquinamento atmosferico sia un fattore di rischio importante associato alle malattie non trasmissibili. Gli effetti dannosi dell'inquinamento sulla salute sono comunemente attribuibili al particolato (PM), una complessa miscela di particelle sospese nell'aria. Il PM può penetrare nel tratto respiratorio inferiore con effetti dannosi diretti e indiretti su diversi organi e tessuti. Gli effetti diretti sono causati dalla capacità dei componenti del PM di attraversare la membrana respiratoria ed entrare nel flusso sanguigno; gli effetti indiretti sono conseguenza sistemica della risposta delle vie aeree locali.

Lavori recenti suggeriscono che il PM possa essere un fattore di rischio indipendente anche per alterazioni del metabolismo scheletrico con conseguente bassa densità minerale ossea e incremento del rischio di sviluppare fratture da fragilità. Le fratture da fragilità determinano gravi conseguenze cliniche che influenzano la qualità della vita, la morbilità e la mortalità dei pazienti affetti. In questa interessante review gli autori valutano i meccanismi alla base dell'ipotizzata associazione tra inquinamento atmosferico, in particolare PM, e alterazioni scheletriche. Sono analizzati quattro possibili meccanismi coinvolti: 1) diversi inquinanti atmosferici possono indurre un'infiammazione sistemica di basso grado, che influenza il metabolismo osseo attraverso un effetto specifico sulle citochine pro-infiammatorie (TNF $\alpha$, TGF-a, IL-1 $\beta$, IL-6 e IL-17), sul differenziamento e attività di osteoblasti e osteoclasti $[1,2]$; 2) alcuni inquinanti, in particolare alcuni gas e composti metallici, possono causare danni ossidativi alle vie aeree e alle cellule ossee; 3 ) gruppi di inquinanti possono agire come interferenti endocrini quando si legano ai recettori nelle cellule ossee, modificandone il funzionamento; e 4) l'inquinamento atmosferico può causare in maniera diretta e/o indiretta una carenza di vitamina D. La caratterizzazione di questi meccanismi potrà definire meglio la fisiopatologia del danno scheletrico indotto dell'inquinamento atmosferico [3], riconoscendo quest'ultimo come ulteriore fattore di rischio modificabile per l'osteoporosi e per le altre patologie metaboliche croniche.

Nota della casa editrice Springer Nature rimane neutrale in riguardo alle rivendicazioni giurisdizionali nelle mappe pubblicate e nelle affiliazioni istituzionali.

\section{Bibliografia}

1. Ran D, Ma Y, Liu W et al (2020) TGF- $\beta$-activated kinase 1 (TAK1) mediates cadmium-induced autophagy in osteoblasts via the AMPK/mTORC1/ULK1 pathway. Toxicology 442:152538

2. Brama M, Politi L, Santini P et al (2012) Cadmium-induced apoptosis and necrosis in human osteoblasts: role of caspases and mitogen-activated protein kinases pathways. J Endocrinol Invest 35(2):198-208

3. Chen YY, Kao TW, Wang CC et al (2020) Association between polycyclic aromatic hydrocarbons exposure and bone turnover in adults. Eur J Endocrinol 182(3):333-341

\footnotetext{
S. Migliaccio

silvia.migliaccio@uniroma4.it

1 Dipartimento di Scienze Motorie, Umane e della Salute, Università "Foro Italico", Roma, Italia
} 